\title{
Zur Theorie der Rauchausbeute*
} oon Klaus M. Meyer-Abich, Tabak Technikum Hamburg

Keith und Newsome (1) haben 1957 beim Abrauchen von Cigaretten Rauchausbeuten R in Abhängigkeit von der Zahl $N$ der vorangegangenen Rauchzüge ermittelt. $\mathrm{Daß} R(\mathrm{~N})$ keine lineare Funktion ist, führen sie auf die relative Verminderung der beim Durchströmen der Tabaksäule durchschnittlich eintretenden Verluste bei großen $N$ zurück. Der $(n+l)$ te Zug findet ja stets bei kleinerer Stummellänge als der n-te Zug statt, so daß die (am Mundstück gemessene) Rauchkonzentration des $(n+1)$ ten Zuges größer ist als die des $n$-ten und $R(N)$ mithin schneller wächst als $N$. Keith und Newsome behaupten, daß die Nichtlinearität der Funktion $R(N)$ ("smoke collected") allein auf diesem Sachverhalt beruhe, und versuchen zu zeigen, daß zwischen der während des Rauchvorgangs insgesamt tatsächlich gebildeten Rauchkondensatmenge ("smoke produced") und der Zugzahl ein linearer Zusammenhang besteht. Das dabei benutzte Verfahren des Übergangs vom „smoke collected" zum "smoke produced" soll im folgenden in theoretischer Hinsicht diskutiert werden. Dabei wird gezeigt, daB die empirischen Ergebnisse von Keith und Newsome eine theoretisch genauere Auswertung wünschenswert erscheinen lassen, als sie in der genannten Arbeit gegeben ist. Außerdem ergibt sich eine neue Größe, die zur Charakterisierung der Füllungseigenschaften des Tabaks in Cigaretten möglicherweise von Wert ist.

Bezeichnet $\rho^{\prime}\left(\lambda^{\prime}\right)$ die Rauchkonzentration, gemessen in $\mathrm{mg} / \mathrm{cm}$ (wegen der Symmetrie der Anordnung kann man sich auf eine Raumkoordinate beschränken) am Ort $\lambda^{\prime}$ in derTabaksäule, so wird beim Weiterströmen des Rauches über einen Weg $d \lambda^{\prime}$ die Konzentrationsabnahme $d \rho^{\prime}$ jedenfalls proportional dem Momentanwert $\rho^{\prime}\left(\lambda^{\prime}\right)$ und proportional $d \lambda^{\prime}$ sein, $d . h$.

$$
d \rho^{\prime}=-\frac{\rho^{\prime}\left(\lambda^{\prime}\right)}{f} d \lambda^{\prime}
$$

$f$ hat die Dimension einer Länge und ist sicher zumindest insofern von der Strömungsgeschwindigkeit des Rauches abhängig, als derjenige Teil der auf dem Weg $d \lambda \lambda^{\prime}$ erfolgenden Abnahme $d \rho^{\prime}$ von $\rho^{\prime}$,

$$
\text { - Eingegangen am 10. Januar } 1963
$$


der auf bloßer Ablagerung infolge der Schwerkraft beruht, bei kleinen Geschwindigkeiten größer sein wird als bei großen. Betrachtet man jedoch zunächst einen kontinuierlichen $Z u g$, so ist $f$ jedenfalls unabhängig von $\lambda^{\prime}$ und aus (I) ergibt sich mit der Umformung

$$
\frac{d \rho^{\prime}}{\rho^{\prime}}=d\left(\ln \rho^{\prime}\right)=-\frac{d \lambda^{\prime}}{f}
$$

durch Integration über einen Weg $\lambda$, auf dem die Rauchkonzentration von einem Ausgangswert $p_{0}(O)$ auf den Wert $\rho(\lambda)$ abnehmen möge,

$$
\begin{gathered}
\int_{\rho_{0}}^{\rho} \frac{\mathrm{d} \rho^{\prime}}{\rho^{\prime}}=\int_{\rho_{0}}^{\rho} \mathrm{d}\left(\ln \rho^{\prime}\right)=\int_{0}^{\lambda}-\frac{\mathrm{d} \lambda^{\prime}}{\mathrm{f}} \\
\ln \rho=-\frac{\lambda}{f}+\ln \rho_{0} \\
\rho=\rho_{0} e^{-\frac{\lambda}{f}}
\end{gathered}
$$

Versteht man unter $\lambda$ nun die momentane Cigarettenlänge, gemessen vom Mundstück aus, so besagt (2), daß die Konzentration $p_{0}$ des am Ort $\lambda$ des Glutkegels ursprünglich erzeugten Rauches auf dem Weg $\lambda$ bis zum Mundstück auf den Wert $\rho_{0} e^{-\frac{\lambda}{f}}$ abfällt. $f$ ist diejenige Strecke, über die $\rho$ bei kontinuierlichem Zug auf den e-ten Teil zurückgeht.

Bei diskontinuierlichem Rauchen wird:

1. insofern eine Abweichung von (2) zu beobachten sein, als nunmehr bei jedem Zug derjenige Teil des gebildeten Rauchkondensats, der sich am Ende des Zuges nach erfolgtem Druckausgleich noch in der Cigarette befindet, durch bloße Ablagerung ausfällt. Dieses Defizit wird um so größer sein, je länger die Cigarette noch ist, mit gegen Null gehender Stummellänge jedoch ebenfalls verschwinden. Auf Grund dieses Sachverhalts ist zu erwarten, daß $p(\lambda)$ bei wachsen$\operatorname{dem} \lambda$ und diskontinuierlichem Zug zunehmend kleinere Werte haben wird, als (2) ergibt.

2. Darüber hinaus muß bei diskontinuierlichem Zug mit einer Modifikation von (2) auf Grund der vorerwähnten Geschwindigkeitsabhängigkeit von $f$ gerechnet werden. Dem Einwand, daß bei diskontinuierlichem Zug $f=f\left(\lambda^{\prime}\right)$ zu setzen ist, die zu Gleichung (2) führende Integration also gar nicht zulässig sei, kann dadurch begegnet werden, daß $\int_{0}^{\lambda} \frac{-\mathrm{d} \lambda^{\prime}}{\mathrm{f}\left(\lambda^{\prime}\right)}$

jedenfalls einen bestimmten Wert haben wird und dieser sich als ein Vielfaches von $\lambda$ ausdrücken lassen wird. Nennt man den betreffenden Faktor " $f$ ", so ist in (2) lediglich $f=f(\lambda)$ anzusetzen, womit die Integration (4) dann freilich nicht mehr vereinbar ist.

Keith und Newsome haben bei diskontinuierlichem Rauchen $p(\lambda)$-Werte ermittelt (Fig. 2 in ihrer Arbeit) und auf einen gradlinigen Verlauf dieser Funktion geschlossen, was formal der unter Absehung von allen Gliedern höheren als ersten Grades geltenden ersten Näherung von (2),

$$
p=p_{0}\left(1-\frac{\lambda}{f}\right)
$$

entspricht. Nun ist ein gradliniger Verlauf der Funktion $\rho(\lambda)$ dort nicht schon deshalb unwahrscheinlich, weil es dann eine Cigarettenlänge gäbe, bei der nur o\% des in der Glutzone gebildeten Rauches aus dem Mundstück austreten würde. Denn bei diskontinuierlichem Rauchen kann es eine solche Länge aus den bei der Diskussion der Anwendbarkeit von (2) auf diskontinuierliches Rauchen angeführten Gründen durchaus geben. Es ist aber höchst unwahrscheinlich, daß dieser Wert unter den Abrauchbedingungen von Keith und Newsome bereits bei $57 \mathrm{~mm}$ liegt, wie dort in Fig. 2. 
Zum anderen scheinen die Meßpunkte von Keith und Newsome weniger auf einer Geraden als vielmehr auf einer Kurve von der Art zu liegen, wie sie vorstehend als zu erwarten geschildert wurde, auf einer Kurve, die sich für kleine $\lambda$ einer Exponentialkurve anschmiegt, für größere $\lambda$ jedoch stärker abfällt als diese. In diesem Zusammenhang ist es nicht ohne Interesse, daß auch die auf "smoke produced" korrigierten Werte sich den bei Keith und Newsome in Fig. 3-6 gezeichneten Geraden nur bis auf einen leichten "Exponentialdrall" anpassen. Ergäbe sich, wenn der bei Keith und Newsome gemäß der Näherung (3) vollzogene Ubergang von "smoke collected" $\mathrm{zu}$ "smoke produced" nunmehr nach der für diskontinuierliches Rauchen passend modifizierten Formel (2) durchgeführt würde, daß die "smoke produced"-Werte auf echten Geraden liegen und keine systematische e-Krümmung mehr aufweisen, so würde nicht nur die obige Theorie, sondern auch die Folgerung von Keith und Newsome erhärtet, daß die "smoke produced"-Werte nicht von der Stummellänge abhängen und $\rho_{0}$ sich mithin mit $\lambda$ nicht ändert.

Allerdings wird sich eine quantitativ genauere Auswertung der bezüglich $\rho(\lambda)$ von Keith und Newsome angestellten Messungen nicht nur wegen der geringen Zahl der dort vorliegenden Meßwerte als mit Schwierigkeiten verbunden erweisen, sondern es bedürfte dazu auch einer eingehenderen mathematischen Diskussion der Modifikation von (2) für diskontinuierliches Rauchen. Auf diese Diskussion soll hier zunächst verzichtet werden, da es zur Prüfung der in Rede stehenden $\lambda$-Unabhängigkeit von $\rho_{0}$ der von Keith und Newsome ausgeführten Ermittlung von $\rho(\lambda)$ gar nicht bedarf. Vielmehr genügt die Ausmessung des Wertverlaufs der eingangs erwähnten Rauchausbeutefunktion $\mathrm{R}$ in Abhängigkeit von der Stummellänge bei kontinuierlichem Abrauchen. Würde nämlich eine Cigarette von der Ausgangslänge $l_{0}$ durch einen kontinuierlichen Zug bis zur Stummellänge 1 ( $\lambda$ ist jetzt Integrationsvariable) abgeraucht, so müßte sich, sofern die Voraussetzungen der Gültigkeit von (2) und der Konstanz von $\rho_{0}$ bezüglich $\lambda$ zutreffen, insgesamt die Rauchausbeute

$$
R\left(l_{0}-1\right)=\int_{1}^{l_{0}} \rho d \lambda=\frac{\rho_{0}}{e^{\frac{l_{o}}{f}}\left(e^{\frac{l_{0}-1}{f}}-1\right)}
$$

ergeben. Zeigte die Ausführung dieses Experiments, daß $R\left(l_{0}-1\right)$ tatsächlich den Verlauf einer Exponentialfunktion hat, so würden dadurch nicht nur die Formel (2) und die Behauptung der Unabhängigkeit der "relativen Rauchausbeute“ $\rho_{0}$ von der Stummellänge bestätigt, sondern man könnte aus den erhaltenen Kurven auch Zahlenwerte von $p_{0}$ und $f$ bezüglich der eingestellten Strömungsgeschwindigkeit des kontinuierlichen Zuges direkt ablesen. Darüber hinaus würde durch Variation der Strömungsgeschwindigkeit gezeigt werden können, in welchem Maße $f$ und gegebenenfalls auch $\rho_{0}$ sich mit der Abrauchgeschwindigkeit verändern.

Angesichts der Tatsache, daß ein kontinuierlicher Abrauchvorgang experimentell nicht ohne Schwierigkeiten realisierbar ist, wäre es schon jetzt nicht ohne Interesse, die Abweichungen der von Keith und Newsome bei diskontinuierlichem Rauchen gewonnenen Meßergebnisse von der Theorie (2), (4) einer näheren Prüfung zu unterziehen. Dazu bedarf es freilich wohl genauerer Informationen, als sie in der genannten Arbeit enthalten sind.

Die Größe $f$ ist eine Art Halbwertslänge bzw. $e^{-1}-$ Wertslänge für die Intensitätsabnahme von Tabakrauch beim Durchströmen einer Cigarette. Die Oberlegung liegt nahe, daß sich diese Größe als Dichtekoeffizient des Tabaks in der Cigarette auf der Suche nach einer das Füllvermögen von Tabak hinreichend charakterisierenden Größe als hilfreich erweisen wird.

\section{ZUSAMMENFASSUNG}

Keith und Newsome haben aus einem 1957 angestellten Experiment gefolgert, daß der Wirkungsgrad der Verbrennung von Tabak in Tabakrauch, die relative Rauchausbeute, nicht vom Abstand zwischen Glutkegel und Mundstück der Cigarette abhängt. Diese Folgerung weist in der bei Keith und Newsome gegebenen Form nicht die theoretische Exaktheit auf, die ihr zu verleihen im Hin- 
blick auf die Relevanz des Ergebnisses wünschenswert wäre. In der vorliegenden Arbeit werden aus der behaupteten Konstanz der relativen Rauchausbeute theoretische Konsequenzen gezogen, deren Prüfung eine zuverlässigere Entscheidung ïber die Richtigkeit des behaupteten Sachverhalts erlaubt. Eine empirische Prüfung dieser Konsequenzen ist wünschenswert.

SUMMARY

In 1957 Keith and Newsome concluded from an experiment that the efficiency of the combustion process, i. e. the relative smoke yield, is independent of the distance between the incandescent zone and cigarette tip. It is shown in the present paper that the results set forth by Keith and Newsome are void of the theoretical accuracy which, in view of the relevance of such results, should perhaps form the basis of interpretation. From the alleged constancy of the relative smoke yield the author of this paper draws theoretical conclusions the verification of which will facilitate, in a reliable way, the decision upon the truth of the said allegation. An empiric examination of the author's conclusions is recommendable.

RESUME

Keith et Newsome ont conclu d'une expérience faite en 1957 que l'efficacité du procédé de combustion du tabac, c'est-à-dire le rendement relatif en fumée, ne dépend pas de l'écart entre la zone incandescente et l'embouchure de la cigarette. Cette conclusion telle que Keith et Newsome l'ont exposée ne présente pas l'exactitude théorique qui paraît être souhaitable en considération de l'importance des résultats. Dans le présent travail l'auteur déduit des conséquences théoriques de la constance prétendue du rendement en fumée la vérification desquelles facilitera le jugement sur l'exactitude des faits prétendus. Un examen empirique des conclusions de l'auteur est recommendable.

LITERATUR

1. Keith, C. H. und Newsome, J. R.: Tobacco Science I (1957) 58.

Weitere Hinweise bei:

2. Dobrowsky, A.: Tobacco Science IV (1960) 126.

3. Fordyce, W. B., Hughes, I. W. und Ivinson, M. G.: Tobacco Science V (1961) 70. 